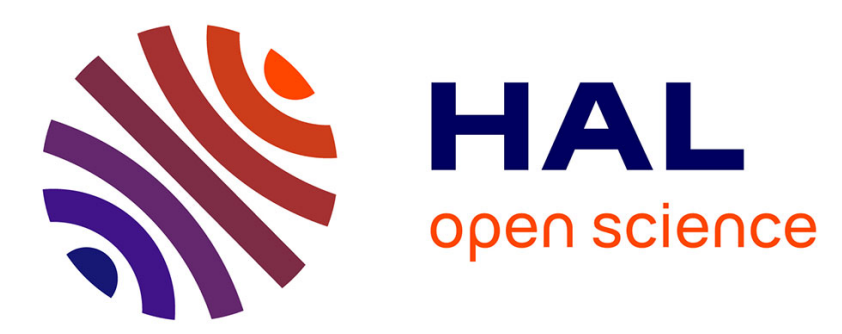

\title{
Inverse identification of a higher order viscous parameter of rigid porous media in the high frequency domain
}

\author{
Rémi Roncen, Zine El Abiddine Fellah, Estelle Piot, Frank Simon, Erick
}

Ogam, Mohamed Fellah, Claude Depollier

\section{- To cite this version:}

Rémi Roncen, Zine El Abiddine Fellah, Estelle Piot, Frank Simon, Erick Ogam, et al.. Inverse identification of a higher order viscous parameter of rigid porous media in the high frequency domain. Journal of the Acoustical Society of America, 2019, 145 (3), pp.1629-1639. 10.1121/1.5095403 . hal02133874

\section{HAL Id: hal-02133874 \\ https://hal.science/hal-02133874}

Submitted on 20 May 2019

HAL is a multi-disciplinary open access archive for the deposit and dissemination of scientific research documents, whether they are published or not. The documents may come from teaching and research institutions in France or abroad, or from public or private research centers.
L'archive ouverte pluridisciplinaire HAL, est destinée au dépôt et à la diffusion de documents scientifiques de niveau recherche, publiés ou non, émanant des établissements d'enseignement et de recherche français ou étrangers, des laboratoires publics ou privés. 


\title{
Inverse Identification of a Higher Order Viscous Parameter of Rigid Porous Media in the High Frequency Domain
}

\author{
R. Roncen ${ }^{* 1}$, Z.E.A. Fellah ${ }^{2}$, E. Piot ${ }^{1}$, F. Simon ${ }^{1}$, E. Ogam ${ }^{2}$, M. Fellah ${ }^{3}$ and C. Depollier ${ }^{\dagger 4}$ \\ ${ }^{1}$ ONERA / DMPE, Université de Toulouse, F-31055, Toulouse, France \\ ${ }^{2}$ LMA, CNRS, UMR 7031, Aix-Marseille Univ, Centrale Marseille, F-13402 Marseille Cedex 20, France \\ ${ }^{3}$ Laboratoire de Physique Théorique, Faculté de Physique, USTHB, BP 32 El Alia, Bab Ezzouar 16111, Algeria \\ ${ }^{4}$ MPEI, Krasnokazarmennaya 14 Moscow 111250, Russia
}

\begin{abstract}
In this paper, a modeling extension for the description of wave propagation in rigid porous media at high frequencies is used. To better characterize the visco-inertial and thermal interactions between the fluid and the structure in this regime, two additional characteristic viscous and thermal surfaces $\Sigma$ and $\Sigma^{\prime}$ are taken into account, as initially introduced in [J. Kergomardet al., Acta Acust united Ac 99 (4) (2013) 557-571]. This extends the modeling order of the dynamic tortuosity and compressibility. A sensitivity analysis is performed on the additional parameters, showing that only the viscous surface $\Sigma$ has an influence on transmitted waves in the high frequency regime, for materials having a low viscous characteristic length. A general Bayesian inference is then conducted to infer simultaneously the posterior probability densities of the parameters associated with the visco-inertial effects, i.e., the porosity, tortuosity, the viscous characteristic length, and the viscous characteristic surface. The proposed method is based on the measurement of waves transmitted by a slab of rigid porous material in the time domain. Bayesian inference results obtained on three different porous materials are presented.
\end{abstract}

\section{Introduction}

The study of the propagation of sound in rigid porous media has found a widespread interest in the acoustic community, due to the different fields of applications in which porous materials are found: sound attenuation [1, hydrology [2], medicine (cancellous bone [3]) and geophysics [4] being some of the most active fields. The knowledge of the intrinsic micro-structural properties intervening in the models used to describe the acoustic behavior of such a medium (i.e., porosity, tortuosity, etc.) is of prime importance, and its pursuit has met wide interest in the literature (for a review, see Refs. [5, 6]).

The measurement of these properties has been tackled with two different classes of methods in the past: direct (noniterative, analytical) and inverse (iterative) methods. Direct methods rely on the use of a straightforward analytical relationship between a measured signal and a model. Early examples of such an approach are found in Refs. [7, 8, where the porosity and flow resistivity of a porous sample are both simply related to a change of volume and pressure loss across the sample, respectively. Recent examples of direct methods include Refs. [9, 10, 11, 12, where a direct relationship is used for the analytical reconstruction of the intrinsic parameters, from an acoustic signal and the Johnson-Champoux-AllardLafarge (JCAL) model [13, 14, 15. Inverse methods rely on an iterative approach, where the difference between the model and experimental signals is minimized. Solutions of different inverse problems for parameter identifications have been attempted regarding air-saturated porous media, mostly in the audible frequency regime [16, 17, 18, 19, 20]. However, the parameters of interest are defined in either the low- or high-frequency asymptotic domain. For porous media, the high frequency domain corresponds to the range of frequencies where the viscous and thermal boundary layer thicknesses become small compared with the curvature radius of the pores [14. Ultrasonic techniques [21, 22, 23, have proven to be efficient in retrieving some of the parameters used to describe the attenuation and dispersion of acoustic waves in a porous medium at high frequencies.

The measurement of ultrasonic reflected waves at the first interface at different oblique incidences was first used to calculate the porosity and tortuosity [9, 24] in a direct manner. Ultrasonic transmitted waves were used to measure the viscous characteristic length [25], and both the viscous and the thermal characteristic lengths [21]. To then extend the identification to more parameters, the ultrasonic reflected and transmitted waves were combined to extract information

\footnotetext{
${ }^{*}$ Corresponding author: remi.roncen@onera.fr

${ }^{\dagger}$ Permanent adress: LUNAM Universite du Maine, UMR CNRS 6613 Laboratoire d'Acoustique de l'Universite du Maine UFR STS Avenue O. Messiaen 72085 Le Mans CEDEX 09, France
} 
on the porosity, tortuosity and the characteristic lengths [23, 11, 26]. While the previous identification attempts were deterministic, Chazot et al. 27] developed a statistical Bayesian approach to identify the properties of the JCA-Biot model [4, relying on frequency-domain signals in the audible regime. This showed the possibility of taking into account the uncertainty of the measurement during the identification procedure. A similar Bayesian approach was then followed [28], where ultrasonic reflected signals from the first interface were used to retrieve the posterior probability densities of the porosity, tortuosity and the viscous and thermal characteristic lengths, thus extending the work presented in Ref. [9].

The recent work of Kergomard et al. 29] has introduced additional terms in the series expansions of the dynamic tortuosity and compressibility used to describe the acoustic behavior of rigid porous media. The associated additional parameters, $\Sigma$ and $\Sigma^{\prime}$, of the same dimension of a surface, have yet to be measured experimentally. The main purpose of this work is to observe the influence of $\Sigma$ and $\Sigma^{\prime}$ on the acoustic response of porous media, and to measure them. An experiment is devised to retrieve information on these additional parameters, using ultrasonic waves transmitted by a slab of rigid porous material and a Bayesian inference process.

The acoustical modeling is first presented in Sec. II. A sensitivity analysis is performed on the additional parameters in Sec. III. In Sec. IV] the Bayesian inference process is summarized, and the inference results are presented for three different materials. Concluding remarks are laid out in Sec. V.

\section{Acoustical Modeling}

When the fluid and solid phases have coupled contributions to the acoustic attenuation in the material, the Biot model [30] is used to describe the wave propagation in both the solid and fluid phases. This results in the occurrence of two compressional waves and one rotational wave. However, the solid structure can often be assumed rigid and its vibrations neglected, especially when air is considered for the fluid phase filling the pores. This leads to the use of an equivalent fluid model where only the compressional wave propagation in the fluid phase of the porous material is considered. The classical properties of density and compressibility are replaced by equivalent ones in order to take into account visco-inertial and thermal fluid-structure interactions in the pores.

\section{A Acoustical high frequency modeling for rigid porous media}

Let us consider a macroscopically homogeneous rigid porous material filled with a fluid of density $\rho_{f}$, fluid adiabatic bulk modulus $K_{f}$ and dynamic viscosity $\eta$. We further assume that the geometry is simple enough so that the movement of the fluid remains incompressible at the pore scale. One can then apply continuum mechanics to the fluid phase in the material and identify the whole porous medium as an equivalent fluid material of effective properties to account for viscous and thermal dissipation arising from the fluid-frame interaction. As initially proposed by Zwikker and Kosten [31, one can decouple the contribution of viscous and thermal dissipation in the pores. To do so, one defines two response factors that represent the deviation from fluid behavior in free space as a function of frequency: the dynamic tortuosity $\alpha(\omega)$ that encompasses all viscous and inertial effects, and the dynamic compressibility $\beta(\omega)$ that takes into account all thermal effects. The equations controlling the wave behavior are given in the frequency domain by

$$
\rho_{f} \alpha(\omega) \mathrm{j} \omega \hat{\boldsymbol{v}}=-\nabla \hat{p}, \quad \frac{1}{K_{f}} \beta(\omega) \mathrm{j} \omega \hat{p}=-\nabla \cdot \hat{\boldsymbol{v}} .
$$

In the previous equations, $\hat{\boldsymbol{v}}$ is the Fourier coefficient of the macroscopic fluid velocity and $\hat{p}$ the Fourier coefficient of the acoustic pressure, obtained by averaging the microscopic velocity and pressure fields over a representative elementary volume. Historically, models are developed in the frequency domain. While the inverse method in this work is conducted using time-domain transmitted signals, frequency-domain models are used to calculate the transmission coefficient. Hence, only frequency-domain models are presented below. The dynamic thermal compressibility is given by [15, 29]

$$
\beta(\omega)=\gamma-(\gamma-1) / \alpha^{\prime}(\omega),
$$

where $\alpha^{\prime}(\omega)$ is the dynamic thermal tortuosity.

Assuming that the pore-surface interface appears locally plane at high enough frequencies, i.e., meaning that the viscous and thermal boundary layers $\left(\delta=\sqrt{2 \eta \rho_{f} \omega}\right.$ and $\left.\delta^{\prime}=\sqrt{2 \eta \rho_{f} \omega \operatorname{Pr}}\right)$ become small compared with a characteristic radius of curvature of the pore, $\alpha(\omega)$ and $\alpha^{\prime}(\omega)$ can be written as power series of $\delta$ and $\delta^{\prime}$ [29]:

$$
\begin{gathered}
\alpha(\omega)=\alpha_{\infty}+\frac{2 \alpha_{\infty}}{\Lambda}\left(\frac{\eta}{\mathrm{j} \omega \rho_{f}}\right)^{1 / 2}+\frac{3 \alpha_{\infty}}{\Sigma}\left(\frac{\eta}{\mathrm{j} \omega \rho_{f}}\right)+\mathcal{O}\left(\frac{1}{\mathrm{j} \omega}\right)^{3 / 2}, \\
\alpha^{\prime}(\omega)=\alpha_{\infty}^{\prime}+\frac{2 \alpha_{\infty}^{\prime}}{\Lambda^{\prime}}\left(\frac{\eta}{\mathrm{j} \omega \rho_{f} \mathrm{Pr}}\right)^{1 / 2}+\frac{3 \alpha_{\infty}^{\prime}}{\Sigma^{\prime}}\left(\frac{\eta}{\mathrm{j} \omega \rho_{f} \mathrm{Pr}}\right)+\mathcal{O}\left(\frac{1}{\mathrm{j} \omega}\right)^{3 / 2},
\end{gathered}
$$


where $\alpha_{\infty}$ is the tortuosity, $\Lambda$ is the characteristic viscous length, $\Sigma$ is the characteristic viscous surface, $\alpha_{\infty}^{\prime}=1$ is the highfrequency limit of the thermal tortuosity [32], $\Lambda^{\prime}$ is the characteristic thermal length and $\Sigma^{\prime}$ is the characteristic thermal surface. The terms in $\Sigma$ and $\Sigma^{\prime}$ were introduced by Kergomard et al. [29] and extend the Johnson-Champoux-Allard model [13, 14] in the asymptotic high-frequency limit, where previously the development stopped at the order 1 in $\delta$ en $\delta^{\prime}$ (the terms in $\Lambda$ and $\Lambda^{\prime}$ ).

\section{B Link with the JCAPL model}

The Johnson-Champoux-Allard-Pride-Lafarge (JCAPL) model [33, 34, 29, 32] can be used to represent the acoustics of rigid porous media from low to high frequencies [29]. The JCAPL model writes, for the viscous effects:

$$
\begin{gathered}
\alpha_{\mathrm{JCAPL}}(\omega)=\alpha_{\infty}\left(1+\frac{8}{M S_{T}^{2}}(1-q)+q \sqrt{1+\frac{M^{2}}{16 q^{2}} S_{T}^{2}}\right) \\
M=\frac{8 \alpha_{\infty} k_{0}}{\phi \Lambda^{2}}, \quad S_{T}=\Lambda \sqrt{\frac{j \omega \rho_{f}}{\eta}}
\end{gathered}
$$

For the thermal effects, one has:

$$
\begin{gathered}
\alpha_{\mathrm{JCAPL}}^{\prime}(\omega)=1+\frac{8}{M^{\prime} S_{T}^{\prime 2}}\left(1-q^{\prime}+q^{\prime} \sqrt{1+\frac{M^{\prime 2}}{16 q^{\prime 2}} S_{T}^{\prime 2}}\right) \\
M^{\prime}=\frac{8 k_{0}^{\prime}}{\phi \Lambda^{\prime 2}}, \quad S_{T}^{\prime}=\Lambda^{\prime} \sqrt{\frac{j \omega \operatorname{Pr} \rho_{f}}{\eta}}
\end{gathered}
$$

In the previous equations, $k_{0}$ is the static viscous permeability (in $\mathrm{m}^{2}$ ), $q$ is the Pride parameter (denoted $P$ in [34]), $k_{0}^{\prime}$ is the static thermal permeability (in $\mathrm{m}^{2}$ ) and $q^{\prime}$ the thermal counterpart of $q$, introduced by Lafarge [33]. The high-frequency terms $q$ and $q^{\prime}$ are related to low-frequency parameters as

$$
q=\frac{1}{\alpha_{0}-\alpha_{\infty}} \frac{2 k_{0} \alpha_{\infty}^{2}}{\phi \Lambda^{2}}, \quad q^{\prime}=\frac{1}{\alpha_{0}^{\prime}-1} \frac{2 k_{0}^{\prime}}{\phi \Lambda^{\prime 2}}
$$

in order for the JCAPL model to match the low-frequency asymptotic model. In these expressions, $\alpha_{0}$ is the static viscous tortuosity (also coined inertial factor, introduced by Norris [35]), and $\alpha_{0}^{\prime}$ is its thermal counterpart [33], the static thermal tortuosity. The extension of Pride can be removed by setting $q=q^{\prime}=1$, thus yielding the Johnson-Champoux-Allard-Lafarge (JCAL) model. When taking the asymptotic expressions of the full model for the dynamic tortuosities (5) and (7), one obtains

$$
\begin{gathered}
\alpha(\omega)=\alpha_{\infty}+\frac{2 \alpha_{\infty}}{\Lambda}\left(\frac{\eta}{\mathrm{j} \omega \rho_{f}}\right)^{1 / 2}+\frac{\phi(1-q)}{k_{0}}\left(\frac{\eta}{\mathrm{j} \omega \rho_{f}}\right)+\mathcal{O}\left(\frac{1}{\mathrm{j} \omega}\right)^{3 / 2}, \\
\alpha^{\prime}(\omega)=\alpha_{\infty}^{\prime}+\frac{2 \alpha_{\infty}^{\prime}}{\Lambda^{\prime}}\left(\frac{\eta}{\mathrm{j} \omega \rho_{f} \operatorname{Pr}}\right)^{1 / 2}+\frac{\phi\left(1-q^{\prime}\right)}{k_{0}^{\prime}}\left(\frac{\eta}{\mathrm{j} \omega \rho_{f} \operatorname{Pr}}\right)+\mathcal{O}\left(\frac{1}{\mathrm{j} \omega}\right)^{3 / 2} .
\end{gathered}
$$

Assuming that the expressions (9) are exact (which is in general not true, as shown explicitly in [29, 2.3]), the introduced viscous and thermal surface parameters $\Sigma$ and $\Sigma^{\prime}$ are related to the earlier parameters of the Pride -Lafarge model as

$$
q=1-\frac{3 \alpha_{\infty} k_{0}}{\phi \Sigma}, \quad q^{\prime}=1-\frac{3 k_{0}^{\prime}}{\phi \Sigma^{\prime}}
$$

Alternatively, a link between the Pride parameter and the pore size distribution was made in Ref. [36]. It was showed [29. Sec. 2.3] that the broadband frequency model of Pride was not capable of satisfactorily accounting for parameters $\Sigma$ and $\Sigma^{\prime}$. In particular, the model fails to match the acoustic behavior of cylindrical circular pores, which is problematic. As such, we find it more lucid to consider the expressions (3-4), which are exempt from this flaw. In addition, there is no longer any need to relate low-frequency and high-frequency parameters when using (3-4). This emphasizes the need for a new measurement method to retrieve $\Sigma$ and $\Sigma^{\prime}$.

\section{Sensitivity analysis}

In this section, numerical simulations of ultrasonic transmitted waves are run by varying the parameters of a numerical porous material, described acoustically using the modified equivalent fluid theory. First, the experimental apparatus used in the following of this work is given in Sec. A. 


\section{A The transmission coefficient}

Experiments are performed in air with two Ultran NCT202 transducers with center frequencies extending from $150 \mathrm{kHz}$ to $230 \mathrm{kHz}$. Pulses of $400 \mathrm{~V}$ amplitude are provided by a 5052PR Panametrics pulser/receiver. The received signals are amplified to $90 \mathrm{~dB}$ and filtered above $1 \mathrm{MHz}$ to avoid high frequency spurious noise. Electronic interference is removed by performing the average of 1000 acquisitions. The experimental setup is shown in Figure 1.

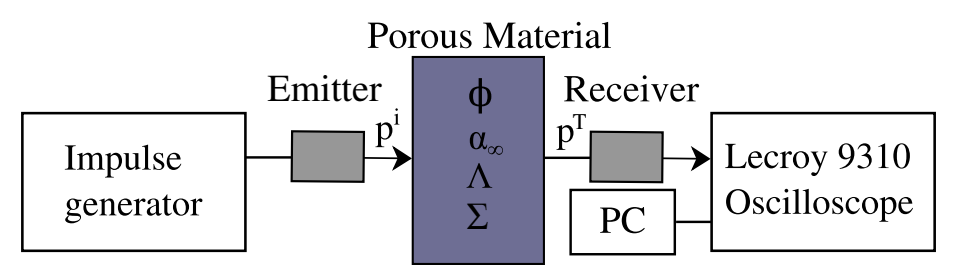

Figure 1: Simplified schematic of the experimental apparatus

When the incident pressure wave is normal to the material, one can obtain the transmission coefficient by solving a system composed of 4 equations obtained by considering continuity of pressure and velocity on each side of the material. Solving the system (see [37, Appendix B]) yields the transmission coefficient $T(\omega)$ in the frequency domain at normal incidence as

$$
T(\omega)=\frac{2 \Xi}{2 \Xi \cosh \left(\mathrm{j} \omega L \xi_{m}\right)+(1+\Xi)^{2} \sinh \left(\mathrm{j} \omega L \xi_{m}\right)},
$$

with $L$ the material width, and

$$
\Xi=\phi \sqrt{\frac{\beta(\omega)}{\alpha(\omega)}}, \quad \xi_{m}=\sqrt{\frac{\rho_{f}}{K_{f}} \alpha(\omega) \beta(\omega)} .
$$

The incident pressure signal $p^{i}(t)$ is Fourier transformed into $\hat{p}^{i}(\omega)$ and multiplied with the transmission coefficient $T(\omega)$. The result is then inverse Fourier transformed to obtain the time-domain transmitted signal as:

$$
p^{T}(t)=\mathrm{iFT}\left[T(\omega) \hat{p}^{i}(\omega)\right],
$$

where iFT is the inverse Fourier transform function, conducted with a Fast Fourier Transform algorithm.

\section{B Parameters sensitivity to the transmitted waves}

A synthetically generated numerical incident pressure is considered. The transmitted pressure associated to the transmission through a certain material is then obtained using Eq. 15. thus mimicking our experiment. The influence of the introduced parameters, i.e., the viscous and thermal surfaces $\Sigma$ and $\Sigma^{\prime}$, is evaluated to see whether there is a chance of identifying them using transmitted signals in the high frequency domain. Figs. 2 5 show the transmitted signals in both the time- and frequency-domain. The signals are obtained with different values of $\Sigma$ and $\Sigma^{\prime}$, for two hypothetical porous materials representative of classical foams encountered in the literature, i.e., a foam P1 with low characteristic viscous length $\Lambda$, and a foam P2 of higher $\Lambda$. The properties of these materials are summarized in Table 1 . In the case

Table 1: Summary of the properties of materials P1 and P2

\begin{tabular}{ccccc} 
Parameters & $\phi$ & $\alpha_{\infty}$ & $\Lambda(\mu \mathrm{m})$ & $\Lambda^{\prime}(\mu \mathrm{m})$ \\
\hline P1 & 0.7 & 1.35 & 50 & 150 \\
P2 & 0.98 & 1.05 & 150 & 450
\end{tabular}

of cylindrical circular pores [29], it is known that $\Sigma=\Sigma^{\prime}=\Lambda^{2}=\Lambda^{\prime 2}=r^{2}$, where $r$ is the pore radius. No other prior knowledge on $\Sigma$ and $\Sigma^{\prime}$ is available yet, since this is the first investigation on the subject, to the best of our knowledge. Thus, as a reference for the sensitivity analysis, we consider that P1 and P2 properties respect the relations

$$
\begin{aligned}
\Sigma & =\Lambda^{2}, \\
\Sigma^{\prime} & =\Lambda^{\prime 2} .
\end{aligned}
$$

These values are then arbitrarily multiplied by a factor 3 to see whether the change has a strong influence on the transmitted signals, for two different frequency contents corresponding to the ones our transducers can produce (centered at around $50 \mathrm{kHz}$ and $200 \mathrm{kHz}$ ). The transmitted signals of the sensitivity analysis are given in Figs. 2 5 . 

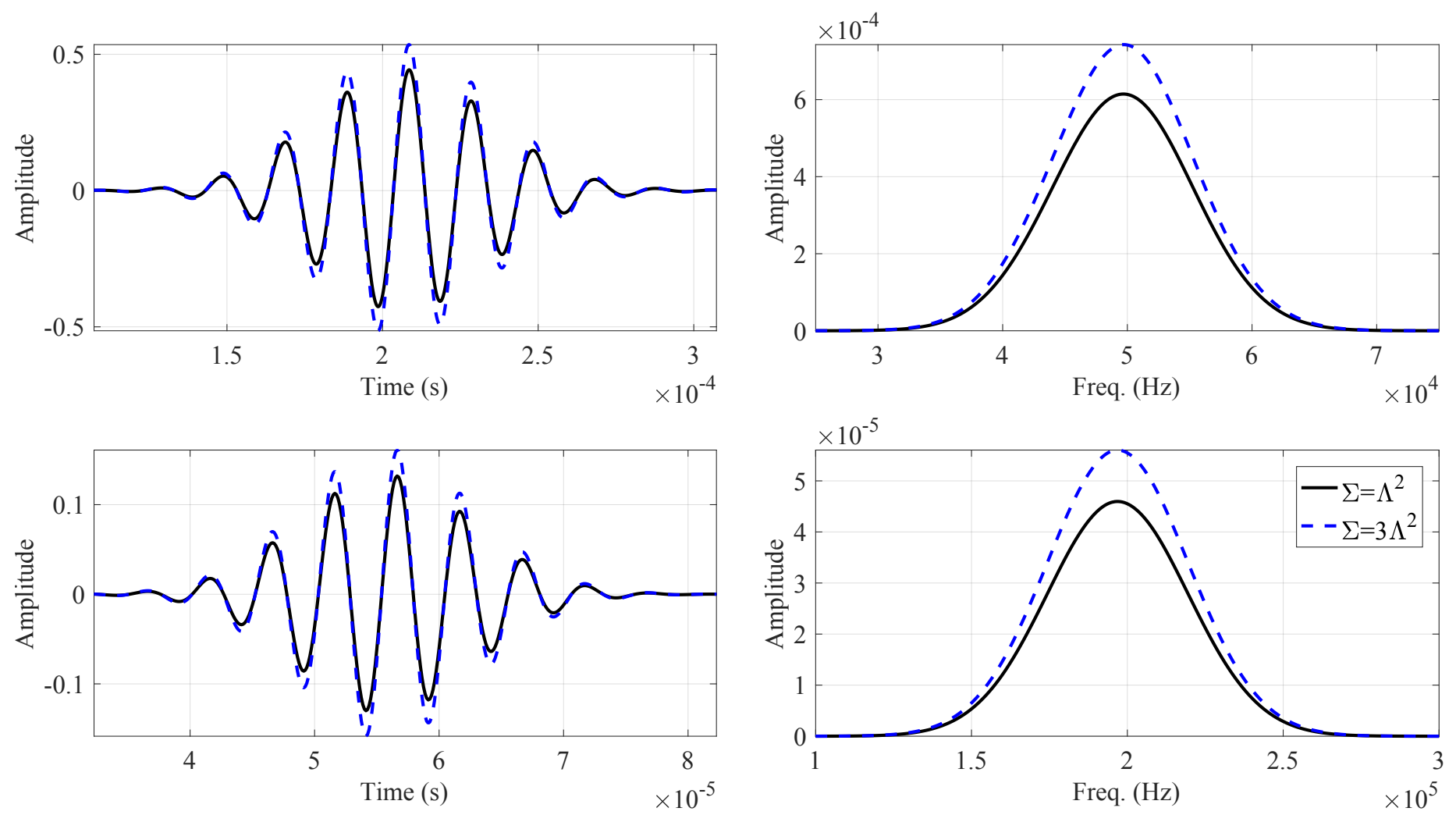

Figure 2: Influence of $\Sigma$ on a transmitted signal in material P1 for two frequency ranges. Upper: [40 - 60] kHz. Lower: $[150-250] \mathrm{kHz}$. Continuous line: $\Sigma=\Lambda^{2}$. Dashed line: $\Sigma=3 \Lambda^{2}$.
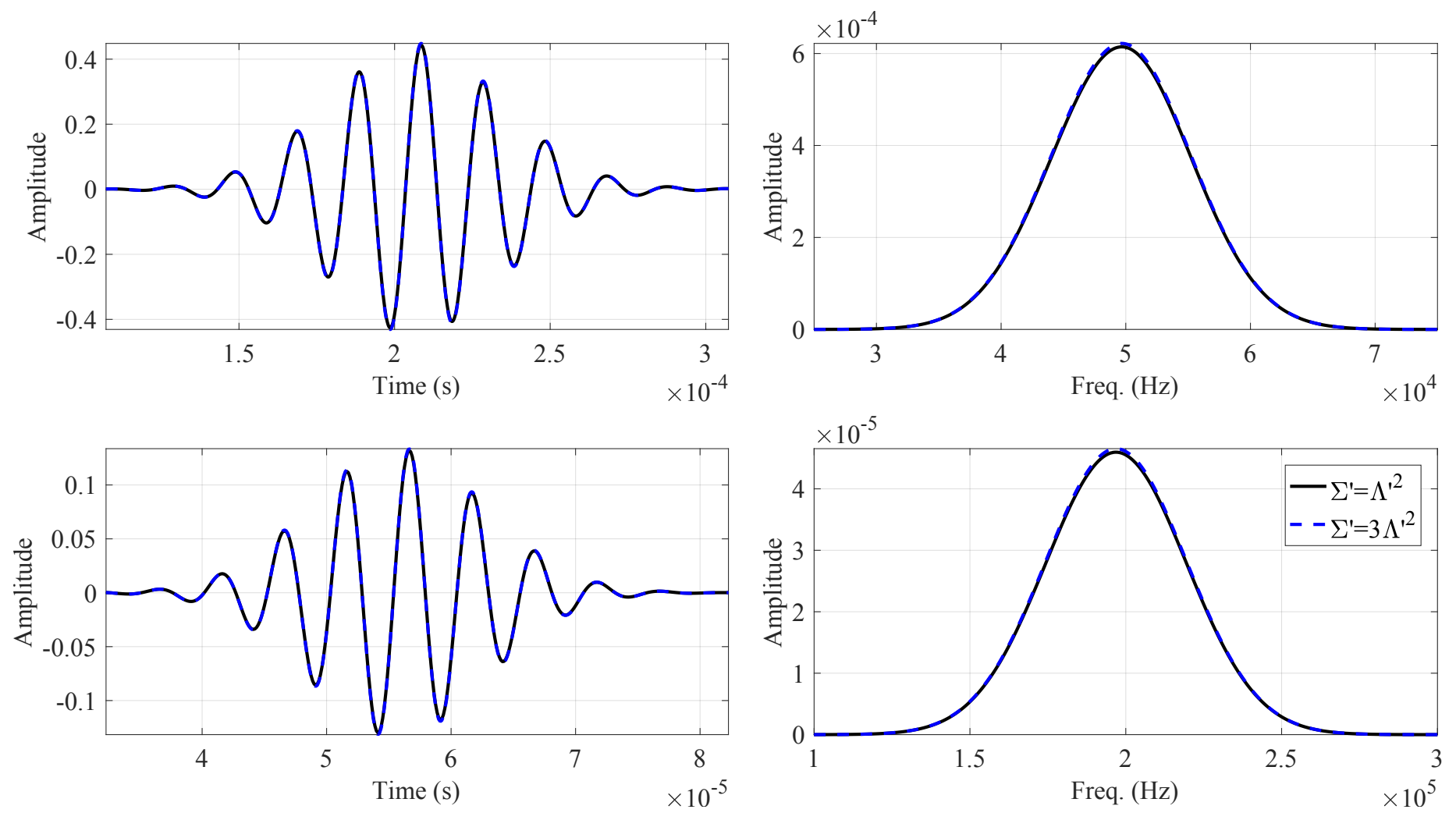

Figure 3: Influence of $\Sigma^{\prime}$ on a transmitted signal in material P1 for two frequency ranges. Upper: [40 - 60] kHz. Lower: $[150-250] \mathrm{kHz}$. Continuous line: $\Sigma^{\prime}=\Lambda^{\prime 2}$. Dashed line: $\Sigma^{\prime}=3 \Lambda^{\prime 2}$. 

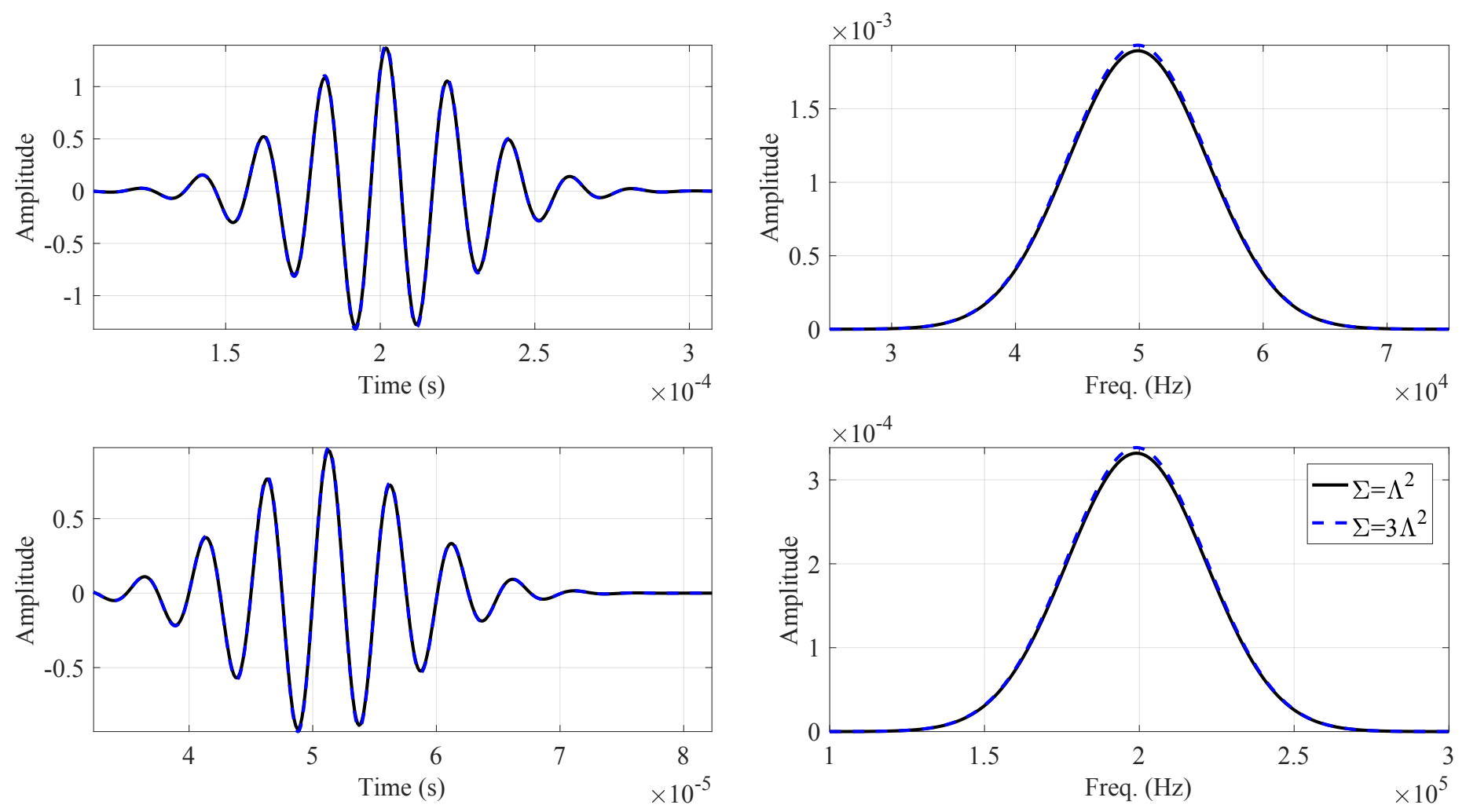

Figure 4: Influence of $\Sigma$ on a transmitted signal in material P2 for two frequency ranges. Upper: [40 - 60] kHz. Lower: $[150-250] \mathrm{kHz}$. Continuous line: $\Sigma=\Lambda^{2}$. Dashed line: $\Sigma=3 \Lambda^{2}$.
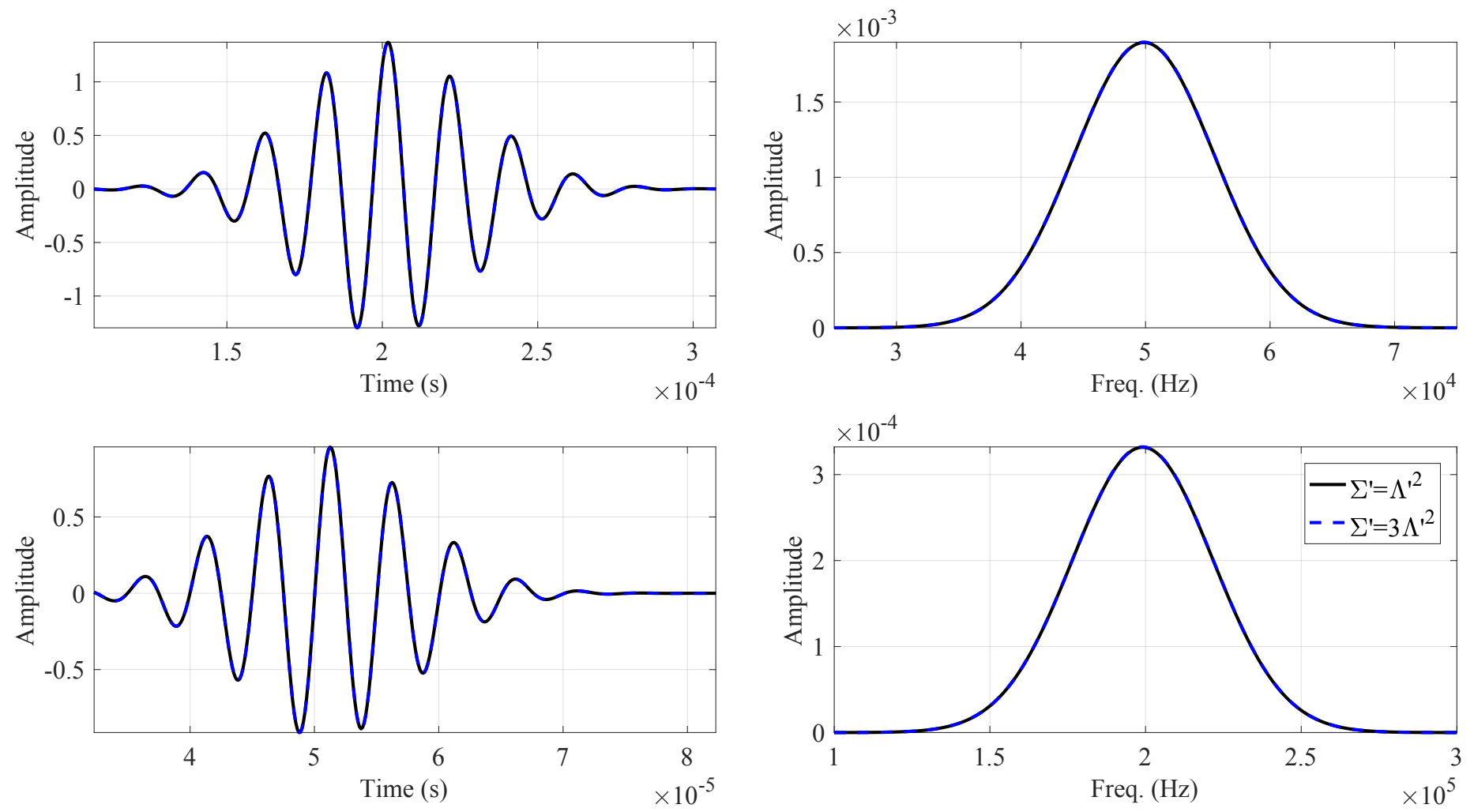

Figure 5: Influence of $\Sigma^{\prime}$ on a transmitted signal in material P2 for two frequency ranges. Upper: [40 - 60] kHz. Lower: $[150-250] \mathrm{kHz}$. Continuous line: $\Sigma^{\prime}=\Lambda^{\prime 2}$. Dashed line: $\Sigma^{\prime}=3 \Lambda^{\prime 2}$.

The influence of $\Sigma$ is mostly visible for resistive materials (low viscous characteristic length). The parameter $\Sigma^{\prime}$ does 
not appear to have any influence on either of the two materials considered. The following will thus focus on materials of low viscous characteristic lengths. The parameter $\Sigma^{\prime}$ is non-influential, it will thus be fixed at $\Sigma^{\prime}=\Lambda^{\prime 2}$ in the remaining of this work. We could also have decided not to consider the term with $\Sigma^{\prime}$ in Eq. 4 instead, which is equivalent to setting $\Sigma^{\prime}=+\infty$. We preferred to give what we thought was a reasonable value of $\Sigma^{\prime}$. Furthermore, we know [21, 38, 22, 25, 23] that the thermal characteristic length $\Lambda^{\prime}$ is usually a factor $2-3$ greater than $\Lambda$, and that its identification is problematic due to a low sensitivity [39, 28]. As a result, $\Lambda^{\prime}$ is set to $3 \Lambda$ in the following, as was done in Refs. [23, 38, and the present work focuses on identifying the visco-inertial parameters.

\section{Statistical inverse problem}

In this section, the principles of Bayesian inference are recalled, and the identification of the properties of three different porous materials is performed.

\section{A Principle of Bayesian inference}

Statistical inference in the Bayesian framework [40, Chap. 8] consists in recasting parameters of interest as random variables. Their probability densities encompass established information one has on the parameters. The information contained in the observed experimental data $\boldsymbol{d}^{\mathrm{e}}$ and the prior knowledge of the estimated parameters $\boldsymbol{m}$ are combined to update one's knowledge on the inferred parameters. In this work, $\boldsymbol{d}^{\mathrm{e}}$ is a collection of measured transmitted signals in the time domain, and $\boldsymbol{m}=\left(\phi, \alpha_{\infty}, \Lambda, \Sigma\right)$. Using Bayes' theorem, a posterior probability density $\pi\left(\boldsymbol{m} \mid \boldsymbol{d}^{\mathrm{e}}\right)$ is defined as

$$
\pi\left(\boldsymbol{m} \mid \boldsymbol{d}^{\mathrm{e}}\right)=\frac{\pi\left(\boldsymbol{d}^{\mathrm{e}} \mid \boldsymbol{m}\right) \pi(\boldsymbol{m})}{\pi\left(\boldsymbol{d}^{\mathrm{e}}\right)}
$$

where $\mathcal{L}(\boldsymbol{m}) \equiv \pi\left(\boldsymbol{d}^{\mathrm{e}} \mid \boldsymbol{m}\right)$ is the likelihood function, $\pi(\boldsymbol{m})$ is the prior density, and $\pi\left(\boldsymbol{d}^{\mathrm{e}}\right)$ is a normalizing constant not calculated in practice. The posterior probability $\pi\left(\boldsymbol{m} \mid \boldsymbol{d}^{\mathrm{e}}\right)$ reflects all the information inferred on $\boldsymbol{m}$, conditional on the observation $\boldsymbol{d}^{\mathrm{e}}$, and is the quantity to be found.

\section{A.1 Likelihood}

The likelihood function represents the mechanism through which new information is assimilated. The physical correlations between $\boldsymbol{m}$ and $\boldsymbol{d}^{\text {e }}$ are represented by a deterministic forward operator $\boldsymbol{G}$ representing the transmission problem, and a noise $\boldsymbol{\epsilon}$ accounting for the uncertainty:

$$
\boldsymbol{d}^{\mathrm{e}}=\boldsymbol{G}\left(\boldsymbol{m}^{*}\right)+\boldsymbol{\epsilon}
$$

where $\boldsymbol{m}^{*}$ is unknown. The components of $\boldsymbol{\epsilon}$ are assumed to be independent of the model parameters $\boldsymbol{m}$ and identically distributed random variables of zero mean Gaussian density $\pi_{\epsilon}$. The forward operator is represented in a discrete-time manner by noting $\boldsymbol{G}(\boldsymbol{m})=\left(G_{1}(\boldsymbol{m}), \ldots G_{j}(\boldsymbol{m}), \ldots, G_{N_{d}}(\boldsymbol{m})\right)$ where $N_{d}$ is the number of time steps observed in the experimental transmitted signal, and with

$$
G_{j}(\boldsymbol{m})=\int_{0}^{t_{j}} \tilde{T}(\boldsymbol{m}, \tau) p^{i}\left(t_{j}-\tau\right) \mathrm{d} \tau
$$

The dependency on the parameters $\boldsymbol{m}$ has been made explicit in the writing of the transmission kernel, and $t_{j}$ is the $j^{\text {th }}$ observed time step. The likelihood takes the form

$$
\mathcal{L}(\boldsymbol{m})=\pi_{\epsilon}\left(\boldsymbol{d}^{\mathrm{e}}-\boldsymbol{G}(\boldsymbol{m})\right)
$$

which reads

$$
\mathcal{L}(\boldsymbol{m})=\prod_{j=1}^{N_{d}} \frac{1}{\sqrt{2 \pi \sigma_{j}^{2}}} \exp \left(-\frac{\left|\boldsymbol{d}^{\mathrm{e}}-G_{j}(\boldsymbol{m})\right|^{2}}{2 \sigma_{j}^{2}}\right),
$$

where $\sigma_{j}^{2}$ denotes the noise variance. The larger the value of $\sigma_{j}^{2}$, the larger the estimated uncertainty on the observation. The value of the standard deviation $\sigma_{j}$ is taken in this work to $2 \%$ of the average of the absolute value of the considered experimental transmitted signal, to encompass our uncertainty in the acquisition chain, the material thickness, the experimental conditions (temperature, pressure, etc.) and the possible presence of gaps and leaks around the samples or the microphone. For numerical reasons, the logarithm of the likelihood is used instead of Eq. 21. 


\section{A.2 Prior model}

The prior probability $\pi(\boldsymbol{m})$ summarizes the information obtained independently of the observation $\boldsymbol{d}^{\mathrm{e}}$. It reflects the user knowledge. A wide-range, non-informative improper homogeneous density on the parameters supports represents well one's ignorance, if no prior knowledge is available, and merely sets boundaries for each parameter. Assuming prior independence between the unknown parameters, we set

$$
\pi(\boldsymbol{m})=\prod_{i=1}^{4} \pi_{i}\left(m_{i}\right)
$$

where a uniform probability $\pi_{i}\left(m_{i}\right) \sim \mathcal{U}\left(m_{i, \min }, m_{i, \max }\right)$ is taken for the priors, with $m_{i, \min }$ and $m_{i, \max }$ the boundaries (see Table 2). Note that it is not expected that the value of $\Sigma / \Lambda^{2}$ is lower than 1 . However, this result has not been made

\begin{tabular}{ccccc}
\multicolumn{4}{c}{ Table 2: Prior boundaries } \\
Parameter & $\phi$ & $\alpha_{\infty}$ & $\Lambda(\mu \mathrm{m})$ & $\Sigma / \Lambda^{2}$ \\
\hline Prior Min & 0.4 & 1.01 & 10 & 0.1 \\
Prior Max & 0.99 & 2 & 300 & 20
\end{tabular}

available in the literature yet, to the best of our knowledge, so it was decided to allow for possibly lower values of $\Sigma / \Lambda^{2}$ during the inference.

\section{A.3 MCMC: posterior sampling}

The posterior probability density defined in Eq. 17 is only known up to a normalizing constant $\pi\left(\boldsymbol{d}^{\mathrm{e}}\right)$, a term difficult to calculate. A technique called Markov Chain Monte Carlo (MCMC) is exploited. A random-walk algorithm is designed to explore the posterior density of interest (usually small compared with the parameter space generated by the prior support) and create a Markov chain whose stationary distribution is the distribution of interest. This is done with a Metropolis-Hasting [41, 42] algorithm for the selection process, consisting in an acceptance/rejection step that creates the transition kernel of the Markov chain. The ratio of probabilities used in the acceptance step cancels the normalizing constant, thus removing the need for calculating it. The reader is referred to [41, 42, 43, 44] for the specifics, including that of the MCMC strategy used in the present article (coined MT-DREAM_ZS [44]).

In the present work, 3 Markov chains are used in parallel, on which $10^{5}$ iterations are performed, with the first $10 \%$ of each chain being discarded as burn-in to allow decorrelation with the initial samples. The chains are initialized by sampling from the prior's support. Convergence is checked a posteriori, through a Gelman-Rubin diagnostic [45] and a visual check of the chain samples.

\section{B Identification results}

The inference method is carried on three different porous materials, denoted by M1, M2 and M3. The foams considered in this work are plastic foams of open porosity, used in sound absorption applications. The Maximum A Posteriori (MAP) estimator is chosen as the reference estimator since it is closest in meaning to the estimates obtained through usual deterministic methods. The MAP estimate $\boldsymbol{m}_{\mathrm{MAP}}$ is defined as

$$
\boldsymbol{m}_{\mathrm{MAP}}=\arg \max _{\boldsymbol{m}} \pi\left(\boldsymbol{m} \mid \boldsymbol{d}^{\mathrm{e}}\right)
$$

The results are summarized in Table 3 for inferences of materials M1, M2, and M3. The $95 \%$ credibility intervals (CI) are also given. In the Bayesian sense, such an interval is interpreted as a $95 \%$ probability that the true value of a given parameter falls within the credible region. Figures 6, 8 and 10 show the probability density functions (pdf, diagonal elements of the matrix plot) and joint-pdfs (off-diagonal elements) inferred on materials M1, M2 and M3, respectively. Obtaining the pdf is achieved through a Kernel density estimation of the Markov-Chains resulting from the MCMC simulations, for each parameter. Finally, the identification is concluded by showing the agreement in time- and frequencydomain between experimental and MAP signals, in Figures 7, 9] and 11, for materials M1, M2 and M3, respectively. The excellent agreement between both signals shows the capability of our model to adequately represent the dissipation mechanisms inside the porous material. It is further noted that the taking into account of $\Sigma$ in the modeling allowed a $\approx 15 \%$ increase of the likelihood (Eq. 21) compared with the classical JCA model. This in turn emphasizes the need to take $\Sigma$ into account when modeling low characteristic lengths porous media in the high frequency regime.

A common feature relative to Figs. 6, 8 and 10 is the display of strong correlations between some parameters. Correlations are seen on a 2D posterior density plot by the presence of a thin ellipse, which major axis direction indicates the correlation sign. A strong positive correlation is present between parameters $\alpha_{\infty}$ and $\Lambda$, while strong negative correlations are evidenced between $\Sigma / \Lambda^{2}$ and all the other parameters. These correlations are model-based, and depend on the 
Table 3: Results summary for the inference of M1, M2 and M3. N/A: non-applicable. Reference values are obtained with the technique developed in Refs. [46, 22, 21.

\begin{tabular}{c|ccccc}
\multicolumn{1}{c}{} & Parameter & $\phi$ & $\alpha_{\infty}$ & $\Lambda(\mu \mathrm{m})$ & $\Sigma / \Lambda^{2}$ \\
\hline \multirow{4}{*}{ M1 } & Ref. value & 0.80 & 1.30 & 43.7 & $\mathrm{~N} / \mathrm{A}$ \\
& Inferred value & 0.76 & 1.31 & 46.7 & 2.52 \\
& $95 \%$ cred. interval & $0.72-0.80$ & $1.30-1.32$ & $45.0-48.5$ & $1.75-3.77$ \\
\cline { 2 - 6 } M2 & Ref. value & 0.75 & 1.45 & 50 & $\mathrm{~N} / \mathrm{A}$ \\
& Inferred value & 0.78 & 1.44 & 31.8 & 6.2 \\
& $95 \%$ cred. interval & $0.76-0.81$ & $1.43-1.44$ & $31.6-32.0$ & $5.5-7.5$ \\
\cline { 2 - 6 } M3 & Ref. value & 0.92 & 1.25 & 50.0 & $\mathrm{~N} / \mathrm{A}$ \\
& Inferred value & 0.77 & 1.24 & 47.0 & 5.7 \\
& $95 \%$ cred. interval & $0.69-0.84$ & $1.23-1.24$ & $46.2-48.6$ & $3.6-18.6$
\end{tabular}
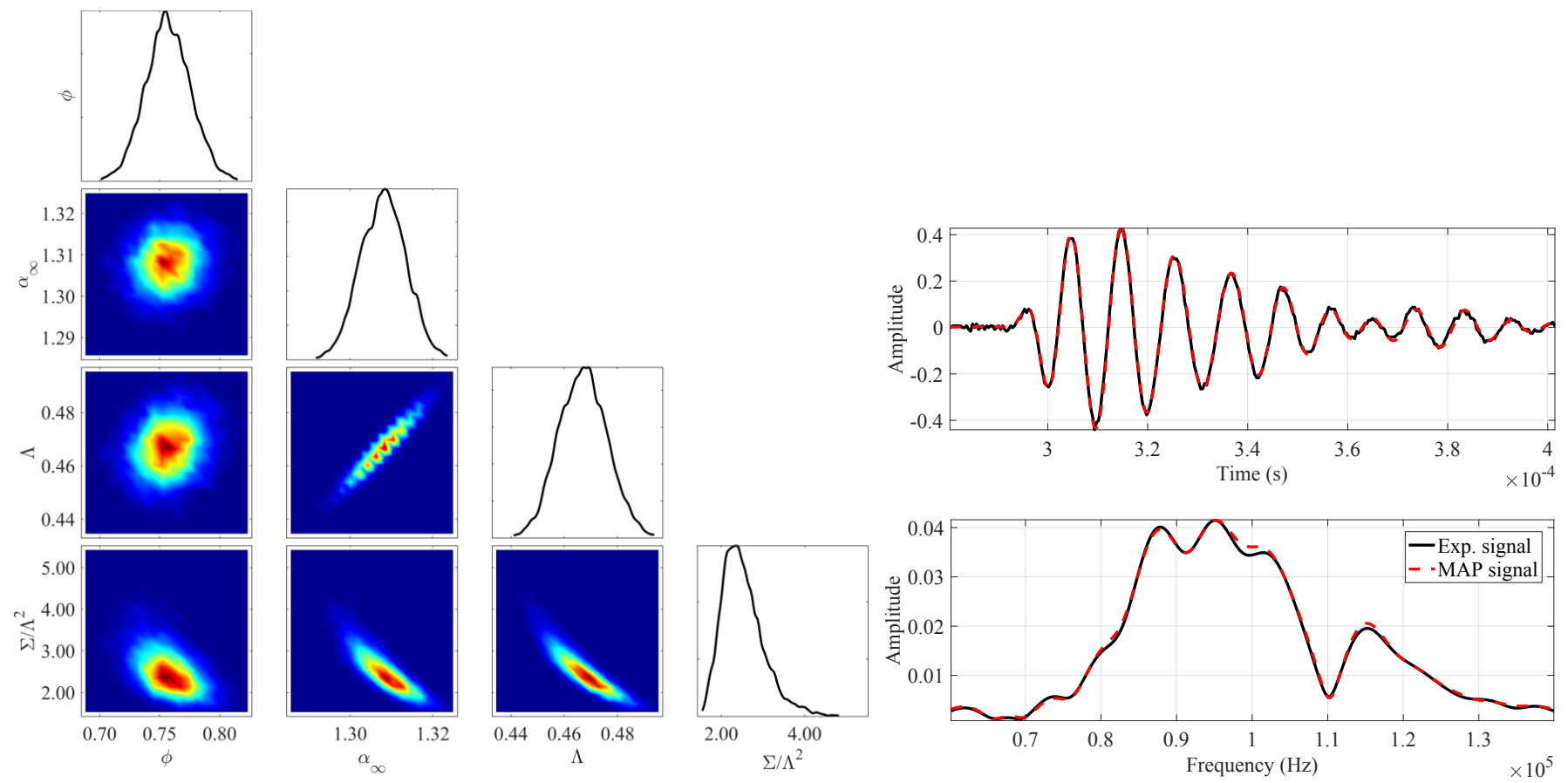

Figure 6: (Color online) M1 inference pdfs (diagonal elements), Figure 7: Material M1. MAP estimates (dashed joint pdfs (off-diagonal elements).

line) and experimental signals (Continuous line). Up: time signals. Down: frequency spectra.

experimental observation that has been made. They are related to the structure of the model used in Eq. 3. For instance, let us consider the real part of the viscous dynamic tortuosity (Eq. 3), which can be written as

$$
\Re(\alpha(\omega))=\alpha_{\infty}\left(1+\sqrt{\frac{2 \eta}{\omega \rho}} \frac{1}{\Lambda}\right) .
$$

If this were the observed quantity, a positive correlation between the $\alpha_{\infty}$ and $\Lambda$ would ensue: an increase in $\alpha_{\infty}$ could be balanced out by an increase in $\Lambda$ in Eq. 24. This would in turn leave the likelihood unchanged. The idea remains the same in the more complicated case of the full problem addressed in this work. In no case should one interpret these correlations as physical ones. In practice, it has been observed that foams with higher tortuosities might have lower characteristic viscous lengths, thus denoting a negative correlation between those two parameters, as opposed to the positive one observed during the inference. The same finding was reported in [28], where a correlation between $\phi$ and $\alpha_{\infty}$ was evidenced when the inference was performed with reflected signals.

Since the viscous surface $\Sigma$ is a higher order term in the asymptotic expansion, it is in some instances less sensitive than the other parameters. However, the presence of well defined posterior density functions shows that information from the transmitted signals was nevertheless extracted and that it was possible to gain knowledge on this parameter.

The sensitivity of $\Sigma$ and its experimental determination show that this viscous surface term should not be neglected. It is necessary to take this new parameter into account for materials having a low characteristic viscous length, i.e., resistive materials. 

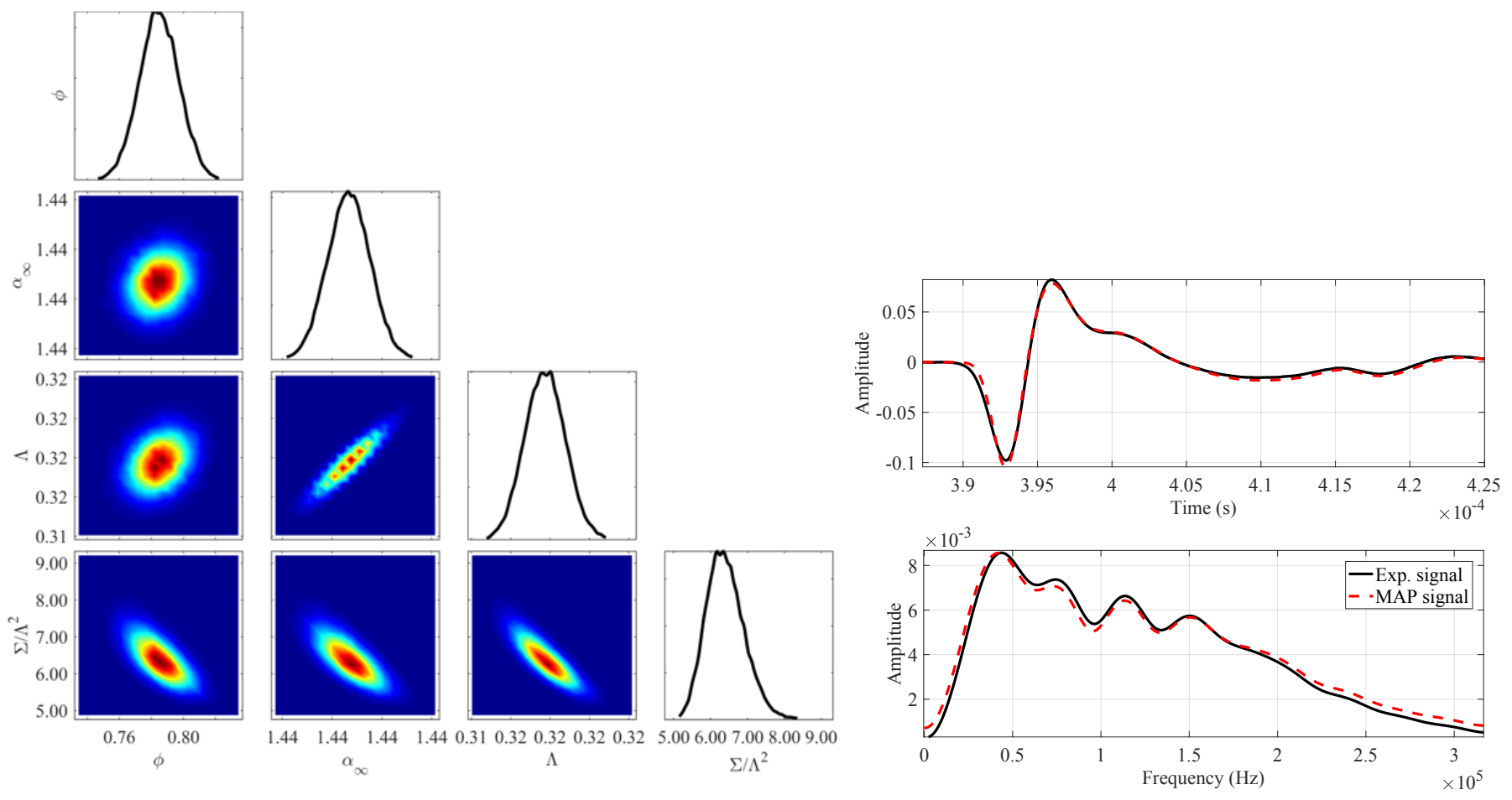

Figure 8: (Color online) M2 inference pdfs (diagonal elements), Figure 9: Material M2. MAP estimates (dashed joint pdfs (off-diagonal elements). line) and experimental signals (Continuous line). Up: time signals. Down: frequency spectra.
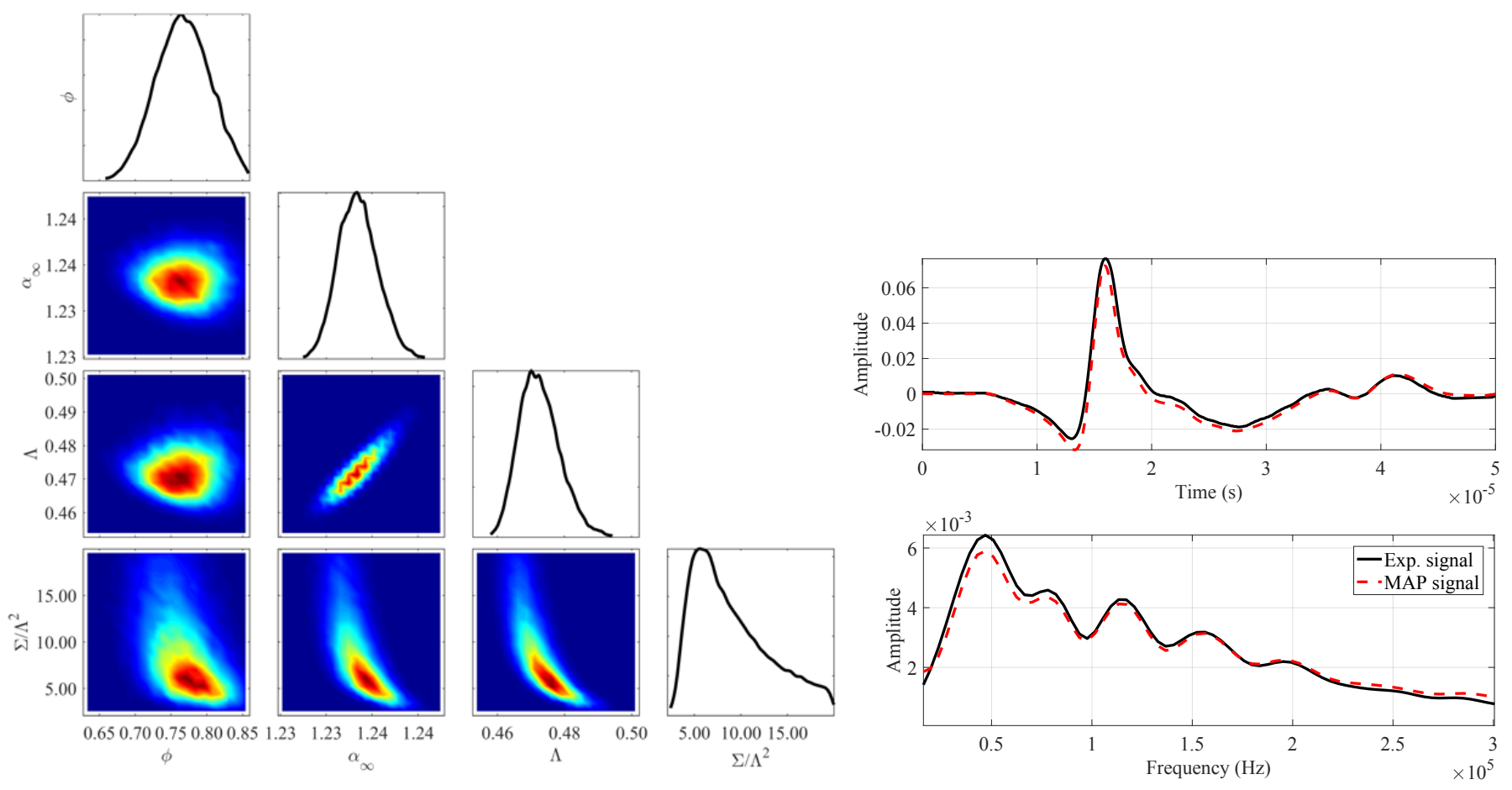

Figure 10: (Color online) M3 inference pdfs (diagonal elements), Figure 11: Material M3. MAP estimates (dashed joint pdfs (off-diagonal elements). line) and experimental signals (Continuous line). Up: time signals. Down: frequency spectra. 


\section{Conclusion}

The modeling of wave propagation inside porous media and the non invasive determination of their micro-structure are crucial for the diverse research fields in which porous materials are studied. In this paper, the propagation of waves through a porous material having a rigid frame is considered in the high frequency regime. This study dealt with two additional parameters intervening in the equivalent fluid model of rigid porous media in the high frequency regime, namely the viscous and thermal characteristic surfaces $\Sigma$ and $\Sigma^{\prime}$. A sensitivity analysis was performed and showed that the viscous surface $\Sigma$ had an influence on the acoustic wave attenuation of ultrasonic waves, but only in porous media with small viscous characteristic lengths. The thermal surface $\Sigma^{\prime}$ was found to have little influence, whatever the porous media.

The study then proposed a statistical inference method based on Bayes theorem to identify the viscous surface $\Sigma$. Ultrasonic transmitted waves were measured in a transmission experiment. No attempt was made in this work to identify the thermal parameters, due to their low expected sensitivity to the transmission problem. The inference process has been carried out on three different porous materials of pores with small cross sections since this is where the influence of the viscous surface $\Sigma$ was predicted to be the greatest.

For the three materials considered, it was possible to extract information from the transmitted wave signals relative to the viscous surface $\Sigma$, which showed a well defined posterior density function. The ratio $\Sigma / \Lambda^{2}$ was consistently identified at values greater than 1 . These identifications warrant additional research to pursue the analytical study of the viscous surface $\Sigma$ and possibly of its thermal counterpart $\Sigma^{\prime}$, even though an experiment has yet to be designed to retrieve its value, possibly involving the use of different gases filling the pores. This study shows the influence and importance of considering the viscous surface $\Sigma$ for acoustic wave attenuation in the high frequency regime, for resistive materials of pores with small cross sections. Therefore, we can no longer ignore this parameter when dealing with wave attenuation in highly resistive porous material.

\section{Acknowledgment}

R.R. would like to thank the French Occitanie region for its financial support. C.D. is supported by the Russian Science Foundation Grant No. 14-49-00079. The authors are grateful for the critical reviews and useful suggestions made by the reviewers.

\section{References}

[1] J. Allard, Modelling sound absorbing materials: Propagation of Sound in Porous Media, Chapman \& Hall, London, 1993.

[2] J. A. Vrugt, C. J. F. ter Braak, M. P. Clark, J. M. Hyman, B. A. Robinson, Treatment of input uncertainty in hydrologic modeling: Doing hydrology backward with markov chain monte carlo simulation, Water Resour. Res. 44 (12), w00B09. doi:10.1029/2007WR006720.

[3] T. Haire, C. Langton, Biot theory: a review of its application to ultrasound propagation through cancellous bone, Bone 24 (4) (1999) 291 - 295. doi:10.1016/S8756-3282(99)00011-3.

[4] M. A. Biot, Mechanics of deformation and acoustic propagation in porous media, J. Appl. Acoust. 33 (4) (1962) 1482-1498. doi:10.1063/1.1728759.

[5] P. Bonfiglio, F. Pompoli, Inversion problems for determining physical parameters of porous materials: Overview and comparison between different methods, Acta Acustica united with Acustica 99 (3) (2013) 341-351. doi:10.3813/ AAA.918616.

[6] K. V. Horoshenkov, A review of acoustical methods for porous material characterisation, Int. J. Acoust. Vib 22 (2017) 92-103. doi:10.20855/ijav.2017.22.1455.

[7] L. L. Beranek, Acoustical properties of homogeneous, isotropic rigid tiles and flexible blankets, J. Acoust. Soc. Am. 19 (4) (1947) 556-568. doi:10.1121/1.1916521

[8] R. L. Brown, R. H. Bolt, The measurement of flow resistance of porous acoustic materials, J. Acoust. Soc. Am. 13 (4) (1942) 337-344. doi:http://dx.doi.org/10.1121/1.1916184.

[9] Z. E. A. Fellah, S. Berger, W. Lauriks, C. Depollier, C. Aristegui, J.-Y. Chapelon, Measuring the porosity and the tortuosity of porous materials via reflected waves at oblique incidence, J. Acoust. Soc. Am. 113 (5) (2003) 2424-2433. doi:10.1121/1.1567275. 
[10] R. Panneton, X. Olny, Acoustical determination of the parameters governing viscous dissipation in porous media, The Journal of the Acoustical Society of America 119 (4) (2006) 2027-2040. doi:10.1121/1.2169923.

[11] J.-P. Groby, E. Ogam, L. De Ryck, N. Sebaa, W. Lauriks, Analytical method for the ultrasonic characterization of homogeneous rigid porous materials from transmitted and reflected coefficients, J. Acoust. Soc. Am. 127 (2) (2010) 764-772. doi:10.1121/1.3283043.

[12] O. Umnova, K. Attenborough, H.-C. Shin, A. Cummings, Deduction of tortuosity and porosity from acoustic reflection and transmission measurements on thick samples of rigid-porous materials, Applied Acoustics 66 (6) (2005) 607-624. doi:10.1016/j.apacoust.2004.02.005.

[13] D. L. Johnson, J. Koplik, R. Dashen, Theory of dynamic permeability and tortuosity in fluid-saturated porous media, J. Fluid Mech. 176 (1987) 379-402. doi:10.1017/S0022112087000727.

[14] Y. Champoux, J.-F. Allard, Dynamic tortuosity and bulk modulus in air-saturated porous media, J. Appl. Acoust. 70 (4) (1991) 1975-1979. doi:10.1063/1.349482.

[15] D. Lafarge, P. Lemarinier, J. F. Allard, V. Tarnow, Dynamic compressibility of air in porous structures at audible frequencies, J. Acoust. Soc. Am. 102 (4) (1997) 1995-2006. doi:10.1121/1.419690.

[16] T. G. Zieliński, Normalized inverse characterization of sound absorbing rigid porous media, J. Acoust. Soc. Am. 137 (6) (2015) 3232-3243. doi:10.1121/1.4919806

[17] Y. Atalla, R. Panneton, Inverse acoustical characterization of open cell porous media using impedance tube measurements, Canadian Acoustics 33 (1) (2005) 11-24.

[18] P. Cobo, F. Simón, A comparison of impedance models for the inverse estimation of the non-acoustical parameters of granular absorbers, J. Appl. Acoust. 104 (2016) 119-126. doi:10.1016/j.apacoust.2015.11.006.

[19] T. Hentati, L. Bouazizi, M. Taktak, H. Trabelsi, M. Haddar, Multi-levels inverse identification of physical parameters of porous materials, J. Appl. Acoust.doi:10.1016/j.apacoust.2015.09.013.

[20] J. Vanhuyse, E. Deckers, S. Jonckheere, B. Pluymers, W. Desmet, Global optimisation methods for poroelastic material characterisation using a clamped sample in a kundt tube setup, Mech. Syst. Sig. Process. 68-69 (2016) 462 - 478. doi:10.1016/j.ymssp.2015.06.027.

[21] P. Leclaire, L. Kelders, W. Lauriks, M. Melon, N. Brown, B. Castagnede, Determination of the viscous and thermal characteristic lengths of plastic foams by ultrasonic measurements in helium and air, J. App. Phys. 80 (4) (1996) 2009-2012. doi:10.1063/1.363817.

[22] N. Brown, M. Melon, V. Montembault, B. Castagnède, W. Lauriks, P. Leclaire, Evaluation of the viscous characteristic length of air-saturated porous materials from the ultrasonic dispersion curve, Comptes rendus de l'Académie des sciences. Série IIb, Mécanique 322 (2) (1996) 122-127.

[23] Z. E. A. Fellah, F. G. Mitri, M. Fellah, E. Ogam, C. Depollier, Ultrasonic characterization of porous absorbing materials: Inverse problem, J. Sound. Vib. 302 (4) (2007) 746-759. doi:10.1016/j.jsv.2006.12.007

[24] Z. E. A. Fellah, S. Berger, W. Lauriks, C. Depollier, P. Trompette, J. Chapelon, Ultrasonic measurement of the porosity and tortuosity of air-saturated random packings of beads, J. Appl. Acoust. 93 (11) (2003) 9352-9359. doi:10.1063/1.1572191.

[25] C. Ayrault, A. Moussatov, B. CastagnÃšde, D. Lafarge, Ultrasonic characterization of plastic foams via measurements with static pressure variations, Applied Physics Letters 74 (21) (1999) 3224-3226. doi:10.1063/1.124112.

[26] Z. E. A. Fellah, M. Sadouki, M. Fellah, F. Mitri, E. Ogam, C. Dépollier, Simultaneous determination of porosity, tortuosity, viscous and thermal characteristic lengths of rigid porous materials, J. App. Phys. 114 (20) (2013) 204902. doi:10.1063/1.4833546.

[27] J.-D. Chazot, E. Zhang, J. Antoni, Acoustical and mechanical characterization of poroelastic materials using a bayesian approach, J. Acoust. Soc. Am. 131 (6) (2012) 4584-4595. doi:10.1121/1.3699236.

[28] R. Roncen, Z. E. A. Fellah, F. Simon, E. Piot, M. Fellah, E. Ogam, C. Depollier, Bayesian inference for the ultrasonic characterization of rigid porous materials using reflected waves by the first interface, The Journal of the Acoustical Society of America 144 (1) (2018) 210-221. doi:10.1121/1.5044423. 
[29] J. Kergomard, D. Lafarge, J. Gilbert, Transients in porous media: exact and modelled time-domain green's functions, Acta Acustica united with Acustica 99 (4) (2013) 557-571. doi:10.3813/AAA.918635.

[30] M. A. Biot, Theory of propagation of elastic waves in a fluid-saturated porous solid. ii. higher frequency range, J. Acoust. Soc. Am. 28 (2) (1956) 179-191. doi:10.1121/1.1908241.

[31] C. Zwikker, C. W. Kosten, Sound absorbing materials, Elsevier, New York, 1949.

[32] J. Allard, N. Atalla, Propagation of Sound in Porous Media: Modelling Sound Absorbing Materials 2e, John Wiley \& Sons, New York, 2009. doi:10.1002/9780470747339.

[33] D. Lafarge, Propagation du son dans les matériaux poreux à structure rigide saturés par un fluide viscothermique: Définition de paramètres géométriques, analogie electromagnétique, temps de relaxation, Ph.D. thesis (1993).

[34] S. R. Pride, F. D. Morgan, A. F. Gangi, Drag forces of porous-medium acoustics, Phys. Rev. B 47 (9) (1993) 4964-4978. doi:10.1103/PhysRevB.47.4964.

[35] A. Norris, On the viscodynamic operator in biot's equations of poroelasticity, J. Wave Mat. Interact 1 (1986) 365-380.

[36] K. V. Horoshenkov, J.-P. Groby, O. Dazel, Asymptotic limits of some models for sound propagation in porous media and the assignment of the pore characteristic lengths, The Journal of the Acoustical Society of America 139 (5) (2016) 2463-2474. doi:10.1121/1.4947540.

[37] R. Roncen, Z. E. A. Fellah, D. Lafarge, E. Piot, F. Simon, E. Ogam, M. Fellah, C. Depollier, Acoustical modeling and bayesian inference for rigid porous media in the low-mid frequency regime, The Journal of the Acoustical Society of America 144 (6) (2018) 3084-3101. doi:10.1121/1.5080561.

[38] A. Moussatov, C. Ayrault, B. Castagnède, Porous material characterization - ultrasonic method for estimation of tortuosity and characteristic length using a barometric chamber, Ultrasonics 39 (3) (2001) 195 - 202. doi:10.1016/ S0041-624X(00) 00062-7.

[39] M. Ouisse, M. Ichchou, S. Chedly, M. Collet, On the sensitivity analysis of porous material models, J. Sound Vib. 331 (24) (2012) 5292-5308. doi:10.1016/j.jsv.2012.07.018

[40] R. C. Smith, Uncertainty quantification: theory, implementation, and applications, Vol. 12, Siam, Philadelphia, 2013.

[41] N. Metropolis, A. W. Rosenbluth, M. N. Rosenbluth, A. H. Teller, E. Teller, Equation of state calculations by fast computing machines, The journal of chemical physics 21 (6) (1953) 1087-1092. doi:10.1063/1.1699114.

[42] W. K. Hastings, Monte carlo sampling methods using markov chains and their applications, Biometrika 57 (1) (1970) 97-109. doi:10.1093/biomet/57.1.97.

[43] W. R. Gilks, S. Richardson, D. Spiegelhalter, Markov chain Monte Carlo in practice, CRC press, New York, 1995.

[44] E. Laloy, J. A. Vrugt, High-dimensional posterior exploration of hydrologic models using multiple-try dream(zs) and high-performance computing, Water Resour. Res. 48 (1) (2012) n/a-n/a, w01526. doi:10.1029/2011WR010608

[45] A. Gelman, D. B. Rubin, Inference from iterative simulation using multiple sequences, Statistical science 7 (4) (1992) 457-472. doi:10.1214/ss/1177011136.

[46] Y. Champoux, M. R. Stinson, G. A. Daigle, Air-based system for the measurement of porosity, J. Acoust. Soc. Am. 89 (2) (1991) 910-916. doi:10.1121/1.1894653 